\title{
Entendido: Um Aplicativo para a Comunidade LGBTQIA+
}

\author{
José Alexsandro do Nascimento Silva1, Guilherme Álvaro Rodrigues Maia \\ Esmeraldo', Robson Gonçalves Fechine Feitosa ${ }^{1}$
}

${ }^{1}$ Instituto Federal de Educação, Ciência e Tecnologia (IFCE) - Campus Crato - Rod. CE 292, KM 15, Bairro Gizelia Pinheiro - 63.115-500 - Crato - CE - Brasil

\author{
alexnascimento.jose@gmail.com, \\ \{guilhermealvaro, robsonfeitosa\} difce.edu.br
}

\begin{abstract}
In Brazil, one LGBTQIA+ person dies every 16 hours. About $20 \%$ of companies do not hire LGBTQIA+. The life expectancy of transgender people is 35 years. Due to the social problems to which this community is subjected, the need for information and promotion of social guidelines aimed at it is perceived. In this context, a survey was carried out with 670 people from 22 Brazilian states, who answered a questionnaire on topics of interest to LGBTQIA+ people. Based on the analysis of the questionnaire responses, this paper describes an application for mobile devices that contemplates, from a social perspective, the approach of themes for the promotion of education, safety, health and employment for this community.
\end{abstract}

\begin{abstract}
Resumo. No Brasil morre uma pessoa LGBTQIA+ a cada 16 horas. Cerca de $20 \%$ das empresas não contratam LGBTQIA+. A expectativa de vida das pessoas transgênero é de 35 anos. Por conta dos problemas sociais aos quais essa comunidade está submetida, percebe-se a necessidade de informação $e$ promoção de pautas sociais voltadas a ela. Nesse contexto, foi realizada uma pesquisa com 670 pessoas de 22 estados brasileiros, que responderam a um questionário sobre temas de interesse das pessoas LGBTQIA+. A partir da análise das respostas do questionário, o presente trabalho descreve um aplicativo para dispositivos móveis que contempla, em uma perspectiva social, a abordagem de temas para a promoção da educação, segurança, saúde e emprego para essa comunidade.
\end{abstract}

\section{Introdução}

Por conta da amplitude da diversidade de gênero e sexual, para fazer referência ao grupo de Lesbicas, Gays, Bissexuais, Transsexuais, Travestis, Queer, Intersexo, Assexual, e outros grupos, utiliza-se a sigla LGBTQIA+. Cada letra da sigla LGBTQIA+ representa um grupo na sociedade que sofre violência por não se adequar àquilo que foi normatizado na sociedade [Brasil e Silva 2020]. As violências diárias às quais os LGBTQIA+ são submetidos afeta toda a sua vida; ficando ainda mais evidente quando analisamos um recorte nos dados das pessoas transexuais, onde a expectativa de vida cai para 35 anos, enquanto a expectativa das pessoas cisgênero e heterossexuais é de 75 anos, segundo [Época e Thomaz 2018].

De acordo com uma pesquisa realizada pela empresa Elancer, $20 \%$ das empresas brasileiras assumiram não contratar pessoas LGBTQIA+, e $11 \%$ delas só contratariam se não fossem para cargos de chefia (pesquisa realizada em 2015, com 2075 recrutadores). Quando analisa-se novamente o recorte para as pessoas transexuais, esse número aumenta drasticamente, por conta do preconceito e alta taxa de evasão escolar das pessoas desse grupo. No ano de 2018, especialistas internacionais dos direitos humanos da Organização das Nações Unidas (ONU) relataram que a discriminação contra as pessoas LGBTQIA+ alimenta uma espiral de violência a qual elas estão sujeitas diariamente; além de criar um ambiente favorável ao processo de exclusão de 
oportunidades em todas as facetas da vida, incluindo o acesso à educação e participação de políticas cívicas, contribuindo para a instabilidade econômica, falta de moradia e precarização da saúde [Organização das Nações Unidas 2018].

Por outro lado, o uso de dispositivos móveis está em constante crescimento. Segundo pesquisa realizada pela Association Global System for Mobile Communications (GSMA), associação de operadoras de redes móveis, até 2025 cerca de $80 \%$ da população mundial vai aderir ao uso constante desses dispositivos. Com isso, a utilização de dispositivos móveis e seus aplicativos estão cada vez mais presentes na vida dos usuários como forma também de acesso a informações em tempo real.

Dessa forma, o objetivo deste trabalho é apresentar um aplicativo para dispositivos móveis, que permita o acesso simplificado de informações relevantes à comunidade LGBTQIA+, ampliando e reforçando a divulgação de informações e oportunidades sobre o tema; ofertando um caminho alternativo para garantiros direitos à educação e emprego para todas e todos. Para isso, este artigo está dividido da seguinte maneira: na Seção 2 apresentam-se alguns trabalhos relacionados; na Seção 3 ilustra-se a metodologia adotada para o desenvolvimento do presente trabalho; a Seção 4 traz uma discussão e análise dos dados coletados na pesquisa e apresenta uma proposta de solução por meio do aplicativo implementado; por fim, na Seção 5, são apresentadas as considerações finais e sugestões de trabalhos futuros.

\section{Trabalhos Relacionados}

[Lemos 2005] relata que nos últimos anos houve uma grande popularização da Internet principalmente por conta dos telefones celulares e das redes de acesso à internet sem fio, dessa forma, realizando transformações sociais e na produção e consumo das informações. Por isso, faz-se importante o levantamento dos aplicativos existentes e os impactos nas práticas sociais e na forma de produzir e consumir informação.

A Tabela 1 apresenta um estudo comparativo entre os principais aplicativos relacionados ao tema do presente trabalho. No estudo, foram consideradas as seguintes métricas de comparação: 1) Disponibilidade: esta métrica trata das plataformas onde o aplicativo está disponível para instalação; 2) Funcionalidades: envolve as principais funções observadas no aplicativo; 3) Público-alvo: recomenda quais faixas etárias são mais indicadas para uso do aplicativo; 4) Número de instalações: dados quantitativos de instações do aplicativo; e 5) Impressões: após uma análise do aplicativo, apresenta-se aqui as principais impressões, que vão desde a aspectos de interface gráfica até utilidade.

Analisando a Tabela 1, em um primeiro momento, é possível observar uma baixa diversidade e quantidade de aplicativos que possuem como público alvo a comunidade LGBTQIA+. Dentre os aplicativos, apenas três não estão disponíveis em ambas as lojas de aplicativo, Google Store e Apple Store. É possível observar que 5 (cinco) aplicativos tratam de interações entre usuários, bate papo ou redes sociais. Por fim, 3 (três) aplicativos abortam a temática jurídica LGBTQIA+. Dos aplicativos listados 5 (cinco) não possuem restrições de acesso por idade. Os aplicativos mais populares são Romeo, Happn e Discord, com mais de 5 (cinco) milhões em número de instalações. Na métrica impressões, na sua maioria, os aplicativos apresentaram boa interface gráfica; e, a maioria não é exclusivamente voltada para a comunidade LGBTQIA+.

Ademais, é possível observar que nenhum dos aplicativos avaliados contemplaram todas as métricas propostas para o estudo comparativo, indicando a necessidade de criação de uma nova solução, a qual é proposta no presente trabalho. As seções a seguir detalham os aspectos metodológicos de desenvolvimento, detalhes da solução proposta e resultados preliminares. 
Tabela 1.Principais aplicativos relacionados ao presente trabalho.

\begin{tabular}{|c|c|c|c|c|c|}
\hline Aplicativo & Disponibilidade & Funcionalidades & Público-Alvo & $\begin{array}{c}\text { No. de } \\
\text { Instalações }\end{array}$ & Impressões \\
\hline Homo Driver & $\begin{array}{l}\text { Google Store e } \\
\text { Apple Store }\end{array}$ & $\begin{array}{l}\text { App de carro similar ao } \\
\text { UBER }\end{array}$ & Livre & $10.000+$ & --- \\
\hline TODXS & $\begin{array}{l}\text { Google Store e } \\
\text { Apple Store }\end{array}$ & $\begin{array}{l}\text { Leis, denúncias, órgãos, } \\
\text { mapa da lgbtfobia, } \\
\text { eventos }\end{array}$ & Livre & $10.000+$ & $\begin{array}{l}\text { App com design } \\
\text { muito agradável e } \\
\text { intuitivo }\end{array}$ \\
\hline $\begin{array}{l}\text { Amino } \\
\text { LGBT }\end{array}$ & $\begin{array}{l}\text { Google Store e } \\
\text { Apple Store }\end{array}$ & $\begin{array}{c}\text { Comunidade e Plataforma } \\
\text { de Bate Papo }\end{array}$ & Livre & $100.000+$ & $\begin{array}{l}\text { Muita poluição } \\
\text { visual }\end{array}$ \\
\hline $\begin{array}{l}\text { Bicha da } \\
\text { Justiça }\end{array}$ & --- & $\begin{array}{l}\text { Startup que representa a } \\
\text { luta pelos direitos da } \\
\text { comunidade LGBTI+, } \\
\text { através de um portal } \\
\text { online, que oferece } \\
\text { assessoria jurídica. }\end{array}$ & Livre & --- & $\begin{array}{l}\text { Plataforma para } \\
\text { assessoria jurídica }\end{array}$ \\
\hline Moovz & Apple Store e APK & Rede Social & $17+$ & --- & $\begin{array}{l}\text { Possui um blog } \\
\text { com os mais } \\
\text { diversos temas }\end{array}$ \\
\hline Romeo & $\begin{array}{l}\text { Google Store e } \\
\text { Apple Store }\end{array}$ & $\begin{array}{c}\text { Relacionamentos, } \\
\text { Encontros e Bate Papo }\end{array}$ & $14+$ & $5.000 .000+$ & $\begin{array}{l}\text { Somente para } \\
\text { homens }\end{array}$ \\
\hline Happn & $\begin{array}{l}\text { Google Store e } \\
\text { Apple Store }\end{array}$ & Encontros & $14+$ & $50.000 .000+$ & $\begin{array}{l}\text { App não utilizado } \\
\text { somente por } \\
\text { LGBTs }\end{array}$ \\
\hline $\begin{array}{l}\text { Te Orienta } \\
\text { Bixa }\end{array}$ & Google Store & Leis e Mapa de Violência & Livre & $1.000+$ & --- \\
\hline Discord & Google Store & Bate Papo & $12+$ & $50.000 .000+$ & $\begin{array}{c}\text { App para bate papo } \\
\text { dos mais diversos } \\
\text { temas }\end{array}$ \\
\hline
\end{tabular}

\section{Metodologia de Pesquisa}

Para o desenvolvimento do presente trabalho, inicialmente realizou-se uma pesquisa do tipo quanti-qualitativa, com o propósito de investigar as percepções, satisfações e expectativas ligadas ao contexto das pessoas LGBTQIA+, possibilitando assim um levantamento de informações, conceitos e necessidades a serem contempladas como requisitos de funcionalidades de um aplicativo para dispositivos móveis voltado a essa comunidade. [Richardson 1989] define que a pesquisa quantitativa é caracterizada pelo método da quantificação, seja na coleta de informações ou no tratamento dessas através de técnicas estatísticas. Quanto à pesquisa qualitativa, [Richardson 1989] diz que a principal diferença da pesquisa qualitativa se dá pela ausência de instrumentos estatísticos para a base de análise do problema, não medindo ou numerando categorias, possibilitando assim interpretações e narrativas individuais, onde o pesquisador participará descrevendo os significados, garantindo assim percepções como resultados.

A coleta de dados é um dos processos mais importantes para a realização da pesquisa, porque é durante essa etapa que obtém-se as informações necessárias para desenvolver um estudo [Oliveira et al. 2016]. No trabalho aqui apresentado, para a realização da coleta de dados, aplicou-se um questionário, entre os dias 30 de julho e 05 de agosto de 2019, por meio da plataforma Google Forms. O questionário foi amplamente divulgado nas redes sociais, com o compartilhamento do link web do formulário para preenchimento individual. Os respondentes do formulário confirmaram participação, aceitando o Termo de Consentimento Livre e Esclarecido (TCLE), onde as informações seriam anonimizadas e utilizadas exclusivamente para fins acadêmicos.

Os dados coletados foram agrupados através das respostas às questões subjetivas e objetivas de caráter qualitativo e quantitativo. Esse método se caracteriza como pesquisa Survey onde a coleta de dados ou informações sobre características ou opiniões 
de determinado grupo de pessoas, população-alvo, é realizada por meio de um instrumento de pesquisa, um questionário, por exemplo [Tanur 1982 apud Pinsonneault e Kraemer 1993].

Após a aplicação do questionário e tabulação dos resultados, realizou-se um comparativo dos principais aplicativos, que tenham como público alvo a população LGBTQIA+, conforme ilustrado na Tabela 1 e detalhado na Seção 2, "Trabalhos Relacionados". Como resultado da análise, notou-se a demanda de implementação de um novo aplicativo.

Para apoiar o processo de desenvolvimento do aplicativo aqui apresentado adotou-se a metodologia de levantamento de requisitos baseado no Rational Unified Process (RUP) [Pressman 2010], onde, após a aplicação do questionário e coleta dos dados foram levantados os requisitos funcionais e não funcionais. A implementação do aplicativo foi desenvolvida com o React Native, que é um framework JavaScript criado pelo Facebook [React 2020]. Ele é compatível com os sistemas operacionais iOS e Android, permitindo a utilização de tecnologias necessárias para o bom funcionamento do aplicativo em ambas as plataformas operacionais para dispositivos móveis. A próxima seção detalha as funcionalidades do aplicativo e traz uma breve discussão sobre os resultados.

\section{Apresentação e Análise dos Resultados}

Com o questionário disponibilizado na presente pesquisa, durante o período de uma semana, foi obtido um total de 670 respostas de pessoas das mais diversas orientações e identidades de gênero. Sobre o perfil dos respondentes, tem-se que: 246 (36,71\%) são do sexo feminino, 5 (2,03\%) são de mulheres trans e travestis; e, 241 (34,68\%) são mulheres cisgenero; já as pessoas do sexo masculino são $374(55,82 \%)$, sendo 16 $(4,27 \%)$ de homens trans; e, $358(51,55 \%)$ homens cisgeneros; tem-se que $50(7,46 \%)$ respondentes que não se encaixam no padrão de binaridade, não se identificando assim com nenhum dos gêneros ou com ambos ao mesmo tempo. A orientação sexual dos participantes é: 259 são gays (38,5\%), 186 bissexuais $(27,76 \%), 106$ héteros $(15,82 \%)$, 69 lésbicas $(10,29 \%)$ e $50(7,46 \%)$ participantes possuem outras orientações sexuais. A faixa etária dos participantes foi dividida em 4 classes, onde 186 respondentes possuem abaixo de 19 anos (27,76\%), 473 na classe entre 20 a 29 anos (70,59\%), 51 pessoas na classe entre 30 a 39 anos $(7,61 \%)$ e na classe acima de 40 anos, 10 respondentes $(1,49 \%)$.

As respostas foram organizadas em uma planilha, onde, quando perguntado se os respondentes "conheciam algum aplicativo voltado para a população LGBTQIA+ que não seja de relacionamentos como o grindr, scruff, tinder", 604 deles $(90,1 \%)$ afirmaram não conhecer e apenas $66(9,9 \%)$ disseram que conheciam outras plataformas. Os participantes da pesquisa também foram questionados a respeito de qual temática principal deveria ser abordada por um aplicativo voltado para a população LGBTQIA+. Os respondentes poderiam selecionar mais de uma opção e sugerir outras; entre as opções mais selecionadas e sugeridas se destacaram: direitos, formação educacional, empregabilidade, segurança, entretenimento e assuntos gerais, como notícias diversas.

Questionou-se também sobre "o que não poderia faltar em um aplicativo para LGBTQIA+"; como a pergunta possuia caráter dissertativo, obteve-se uma diversidade de respostas, onde algumas das mais frequentes foram: o aplicativo deveria incluir uma interface visualmente agradável; deve ser inclusivo; que apresente informações de todos os tipos (e.g. notícias, ofertas de empregos e cursos); que seja motivacional e acolhedor; que a sua utilização seja intuitiva; e, que promova uma interação entre seus usuários. Quando questionados se "as pessoas utilizariam um aplicativo que tratasse de outras temáticas, como por exemplo direitos, saúde e emprego", as respostas evidenciaram que existe demanda dentro do mercado de aplicativos. 
Diante das respostas coletadas, por meio do questionário, e após a análise de possíveis requisitos e funcionalidades, formulou-se o aplicativo intitulado "Entendido". A Figura 1 mostra capturas de tela (screenshots) que apresentam os principais recursos do aplicativo aqui proposto. Ao inicializar o aplicativo "Entendido", o usuário é direcionado para um menu principal, onde ele tem acesso às principais funcionalidades do aplicativo, podendo retornar ao menu a qualquer momento tocando na logo com nome "Entendido". A Figura 1 (a), apresenta a tela do "Menu Principal", que, além de atalhos para os demais recursos do aplicativo, inclui o suporte para leitura de notícias, no qual os usuários podem ter acesso às notícias que são compartilhadas a partir de diferentes mídias, tendo como assunto central a causa LGBTQIA+.

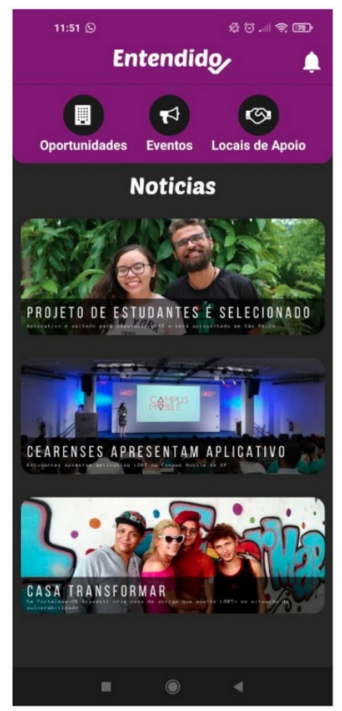

(a) Menu Principal.

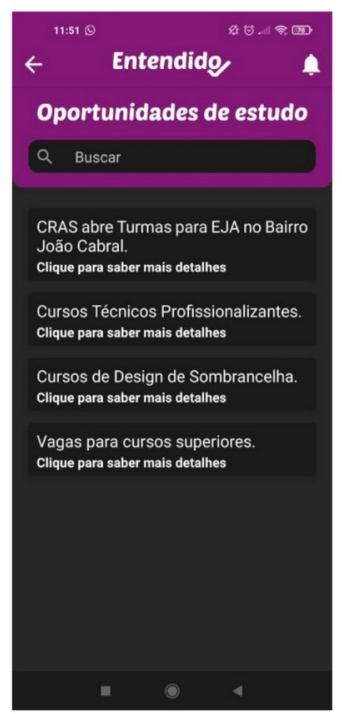

(e) Oportunidades de Estudo.

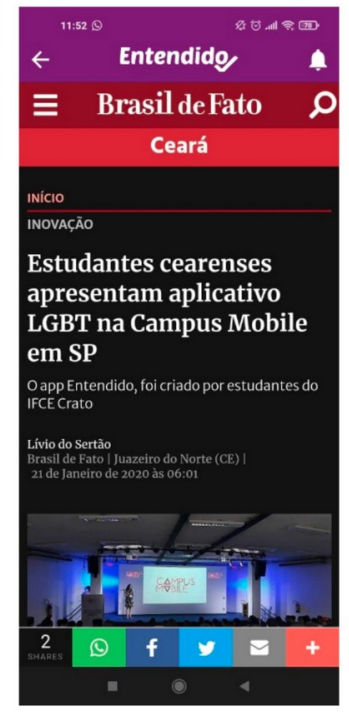

(b) Leitura de Notícias.

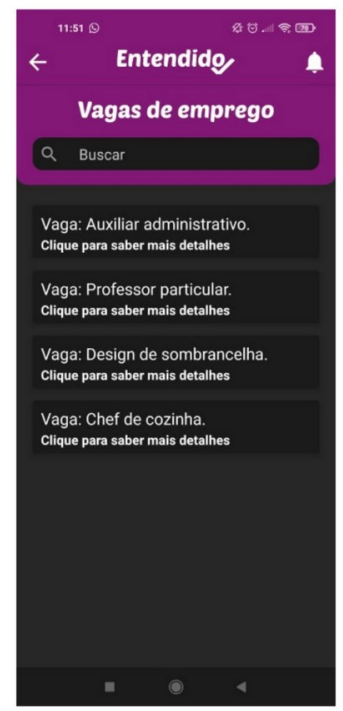

(f) Oportunidades de Emprego.

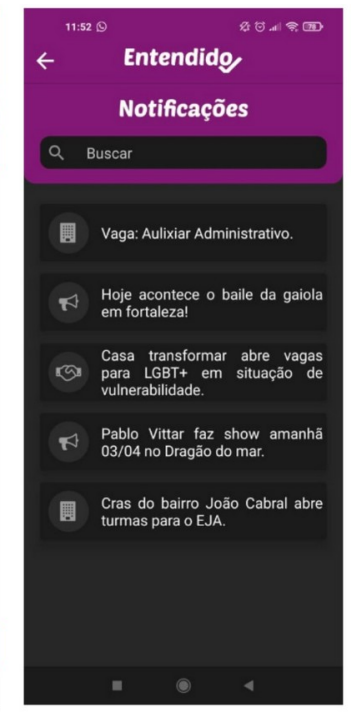

(c) Notificações.

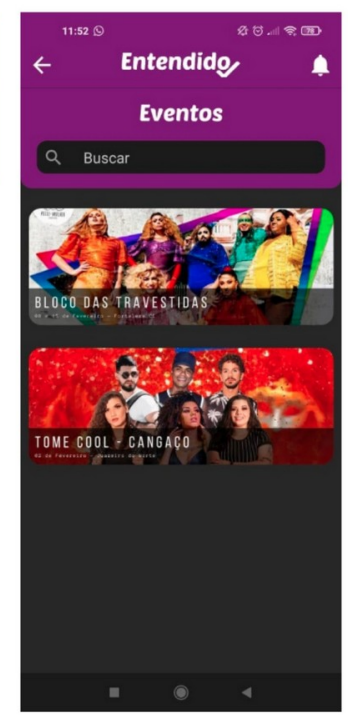

(g) Eventos.

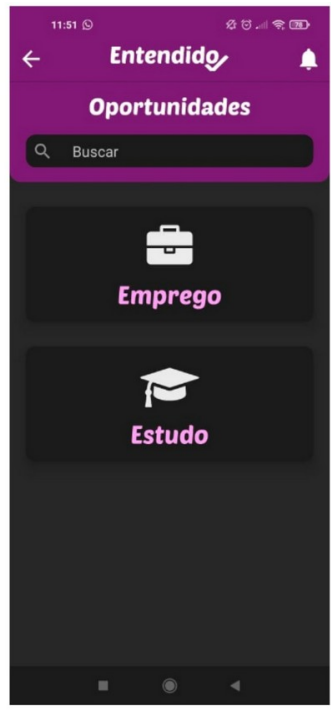

(d) Oportunidades.

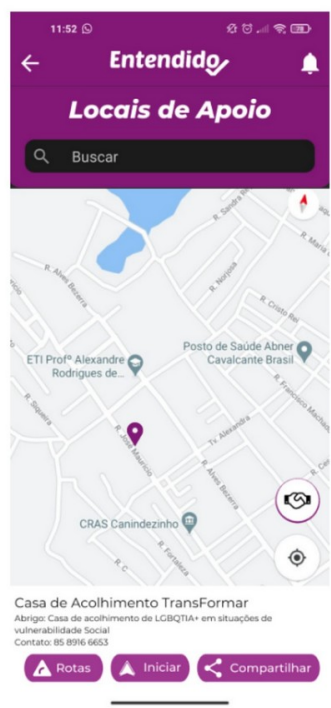

(h) Locais de Apoio.

\section{Figura 1. Interface do aplicativo "Entendido", com apresentação de diferentes} recursos.

Na Figura 1 (b), pode-se observar a tela "Leitura de Notícias", em que, através das notificações, os usuários serão informados quando novas vagas de emprego ou vagas para formação profissional e/ou educacional estarão disponíveis, podendo acessar 
essas informações através da notificação. Na Figura 1 (c), na tela de "Notificações", que faz uso da funcionalidade "Oportunidades", apresentada na Figura 1 (d), os usuários têm acesso a dois novos recursos, que consistem de duas listas, nas quais estão disponíveis vagas de emprego e para formação educacional e profissional. A tela da Figura 1 (e), "Oportunidades de Estudo", trata do acesso dos usuários às informações educacionais de nível básico, médio, superior, profissionalizante e cursos de curta duração. Com o suporte deste recurso, o público alvo pode encontrar locais onde há possibilidade para concluir sua formação acadêmica do ensino médio, obter uma formação profissional ou ingressar no ensino superior.

Já em "Oportunidades de Emprego", como pode ser visto na Figura 1 (f), os usuários tem acesso a informações de vagas de empregos, que não oferecem discriminação por sua orientação sexual, identidade de gênero ou expressão de gênero dentro do convívio e ambiente de trabalho. As vagas disponibilizadas partem de empresas que proeminentemente possuem o compromisso com a diversidade. Na Figura 1 (g), a tela de "Eventos" tem a finalidade de divulgar eventos em que se tenha LGBTQIA+ como seu público alvo; apresenta um mapa cultural de lugares livres de diversos tipos de preconceitos.

Por fim, na Figura 1 (h), "Locais de Apoio" tem a finalidade de construir uma rede de apoio social, de segurança e de saúde, através da localização via GPS do próprio aparelho do usuário, ou mediante busca textual. Com esse recurso, os usuários do aplicativo podem encontrar serviços essenciais, ofertados por profissionais que atendam e que tenham conhecimento das particularidades LGBTQIA+. Entre os registros, podese encontrar médicos e psicólogos, casas de acolhimento - como, por exemplo, a casa Transformar, localizada na cidade de Fortaleza-Ceará -, e demais estabelecimentos que podem contribuir, de diferentes formas, para aumento da qualidade de vida e bem estar dos usuários do aplicativo.

O aplicativo proposto, como pode-se verificar nos parágrafos anteriores, contemplou todos os anseios dos respondentes do formulário da pesquisa de opinião, bem como contempla as principais funcionalidades e impressões das ferramentas da literatura, as quais estão expostas na Tabela 1.

\section{Conclusões}

Este trabalho teve como objetivo contribuir no compartilhamento de informações voltadas exclusivamente para a comunidade LGBTQIA+, de forma a promover sua formação educacional e atuação profissional, bem como oferecer oportunidades de entretenimento e de apoio por profissionais da saúde. Busca-se, desta forma, estabelecer ações que contemplem as diversas faces do ser (social, econômica, política, pessoal, entre outras), com objetivo de possibilitar uma melhor qualidade de vida para as pessoas dessa comunidade.

Compreendendo que a tecnologia por si só, não será a solução dos problemas desta comunidade, a proposta desenvolvida neste trabalho se mostra necessária como forma de combate a marginalização das pessoas LGBTQIA+. Através do aplicativo aqui apresentado, os usuários terão acesso a informações e oportunidades direcionadas ao público-alvo, mostrando que outros caminhos são possíveis e que o direito à educação e emprego são para todas e todos.

O estado atual de maturidade do aplicativo necessita de algumas melhorias, pois atualmente as informações, como as notícias, são exibidas a partir de fontes de sites previamente selecionados, e com a temática LGBTQIA+. Ou seja, será necessário implementar uma ferramenta complementar para auxiliar a atualização dinâmica dos conteúdos, como notícias e oportunidades, por meio dos administradores do sistema. Com o suporte desse novo recurso, os conteúdos poderão ser atualizados de forma mais 
simples, e com a frequência e o volume necessários. Além de dar suporte à curadoria de notícias de fontes externas, o novo sistema poderia permitir que os administradores, junto com uma equipe multidisciplinar, também possam gerar conteúdos diversos e exclusivos.

Outros trabalhos futuros incluem a avaliação dos aspectos de usabilidade e qualidade dos conteúdos do aplicativo "Entendido", a partir do feedback dos usuários em campo; e a inserção de novos recursos, tal como o "botão do pânico", para que o usuário consiga compartilhar sua localização em tempo real com alguém da sua confiança e/ou autoridades policiais, em situações de risco.

\section{Referências}

ABNT - Associação Brasileira de Normas Técnicas. ISO / IEC / IEEE . 29119-1. Engenharia de software e sistemas - Teste de software: Parte 1: Conceitos e definições, [S. 1.], 1 set. 2013. Disponível em: https://www.abntcatalogo.com.br/ norma.aspx?ID=314331. Acesso em: 19 dez. 2019

Adjust. 2019. Adjust Global App Trends 2019: Global Benchmarks Report, [S. 1.], ano 2019, 6 fev. 2019.

Eduardo Michels (Brasil). População LGBT Morta no Brasil: Relatório 2018. Salvador: Grupo Gay da Bahia, 2019. 25 p. Disponível em: <https://grupogaydabahia.files.wordpress.com/2019/01/relat\%C3\%B3rio-de-crimescontra-lgbt-brasil-2018-grupo-gay-da-bahia.pdf>. Acesso em: 15 novembro 2019.

Época (Brasil); Thomaz, Danilo (ed.). Reduzida por homicídios, a expectativa de vida de um transexual no Brasil é de apenas 35 anos: Em 2017, 179 transgêneros foram assassinados no país. [S. 1.]: ÉPOCA, 30 jan. 2018. Disponível em: https://epoca.globo.com/brasil/noticia/2018/01/reduzida-por-homicidios-expectativade-vida-de-um-transexual-no-brasil-e-de-apenas-35-anos.html. Acesso em: 2 nov. 2019.

Filho, Wilson de Pádua Paula. Engenharia de software: fundamentos, métodos e padrões. 602 pg. 2 vl. LCT. São Paulo, 2009.

Gsm Association. The Mobile Economy 2019. In: GSM ASSOCIATION. The Mobile Economy 2019. Https://www.gsma.com/mobileeconomy/, 9 set. 2019. Disponível em: https://www.gsma.com/mobileeconomy/. Acesso em: 26 out. 2020.

Lemos, Andre. Cibercultura e Mobilidade. A Era da Conexão 1. Pesquisa Quantitativa, XXVIII Congresso Brasileiro de Ciências da Comunicação - Uerj, 7 set. 2005.

O Globo (Brasil). Falta de dados oficiais dificulta solução de crimes contra a homofobia. O Globo, 30 Ago. 2018. Disponível em: https://oglobo.globo.com/sociedade/faltade-dados-oficiais-dificulta-solucao-de-crimes-contra-homofobia-1-22317485. Acesso em: 17 dez. 2020.

Oliveira, José Clovis Pereira de et al. O Questionário, o formulário e a entrevista como instrumentos de coleta de dados: vantagens e desvantagens do seu uso na pesquisa de campo em Ciências Humanas. Formulário, Congresso Nacional de Educação, ed. III, 6 out. 2016.

Organização das Nações Unidas (Brasil). Direitos Humanos. População LGBT têm acesso reduzido a direitos sociais, econômicos e culturais, dizem relatores. [S. 1.], 17 maio de 2018. Disponível em: https://nacoesunidas.org/populacao-lgbt-tem-acessoreduzido- a-direitos-sociais-economicos-e-culturais-dizem-relatores/. Acesso em: 20 nov. 2019.

React. Tutorial: Introdução ao React. [S. 1.]. Disponível em: https://pt-br.reactjs.org/tutorial/tutorial.html. Acesso em: 11 dez. 2019. 
Richardson, Roberto Jarry. Pesquisa social: métodos e técnicas. São Paulo: Atlas, 1989. SANTA CATARINA. Proposta Curricular de Santa Catarina. Florianópolis (SC). IOESC, 2005.

Santos e Advogados. O que é diversidade de gênero? Entenda mais sobre o tema. [S. 1.]: Santos Advogados Associados, 8 out. 2019. Disponível em: https://blog.santosadvogadosassociados.com/diversidade-de-genero/. Acesso em: 24 fev. 2021.

Sommerville, Ian. Engenharia de software. 9 ed. São Paulo: Pearson, 2011.

Tanur, J.M. Advances in methods for large-scale surveys and experiments, inR. Mcadams, N.J. Smelser, \& D.J. Treiman (eds.), Behavioral and Social Science Research: A National Resource, Part II. Washington, D.C.: National Academy Press. 1982. 